\title{
Roles of fatty acid metabolism in tumourigenesis: Beyond providing nutrition (Review)
}

\author{
XIANG-HUA YU ${ }^{1 *}$, XIAO-HUA REN ${ }^{2 *}$, XIN-HUA LIANG ${ }^{1}$ and YA-LING TANG ${ }^{3}$ \\ ${ }^{1}$ Department of Oral and Maxillofacial Surgery, State Key Laboratory of Oral Diseases and National Clinical Research Center for \\ Oral Diseases, West China Hospital of Stomatology (Sichuan University), Chengdu, Sichuan 610041; ${ }^{2}$ Department of Stomatology, \\ Sichuan Academy of Medical Sciences and Sichuan Provincial People's Hospital, School of Medicine, \\ University of Electronic Science and Technology of China, Chengdu, Sichuan 610072; ${ }^{3}$ Department of Oral Pathology, \\ State Key Laboratory of Oral Diseases and National Clinical Research Center for Oral Diseases, \\ West China Hospital of Stomatology (Sichuan University), Chengdu, Sichuan 610041, P.R. China
}

Received April 21, 2018; Accepted September 19, 2018

DOI: $10.3892 / \mathrm{mmr} .2018 .9577$

\begin{abstract}
Fatty acid (FA) metabolism, including the uptake, de novo synthesis and oxidation of FAs, is critical for the

Correspondence to: Professor Xin-Hua Liang, Department of Oral and Maxillofacial Surgery, State Key Laboratory of Oral Diseases and National Clinical Research Center for Oral Diseases, West China Hospital of Stomatology (Sichuan University), 14, Sec. 3. Renminnan Road, Chengdu, Sichuan 610041, P.R. China

E-mail: 1xh88866@scu.edu.cn; drliangxinhua@aliyun.com
\end{abstract}

Professor Ya-Ling Tang, Department of Oral Pathology, State Key Laboratory of Oral Diseases and National Clinical Research Center for Oral Diseases, West China Hospital of Stomatology (Sichuan University), 14, Sec. 3, Renminnan Road, Chengdu, Sichuan 610041, P.R. China

E-mail: tangyaling@scu.edu.cn

*Contributed equally

Abbreviations: FA, fatty acid; FASN, fatty acid synthase; VEGF, vascular endothelial growth factor; VEGFR2, vascular endothelial growth factor receptor 2; FAO, fatty acid oxidation; ECs, endothelial cells; CPT1, carnitine palmitoyl transferase 1; PGE2, phenyl glycidyl ether 2; FABP, fatty acid-binding protein; PPAR $\gamma$, peroxisome proliferator activated receptor $\gamma$; EMT, epithelial-mesenchymal transition; acetyl-CoA, acetyl coenzyme A; FAT, fatty acid transporter; FFA, free fatty acid; SCD1, stearoyl-CoA desaturase-1; ACS, acetyl-CoA synthase; HIF, hypoxia-inducible factor; ACL, ATP citrate lyase; HCC, hepatocellular carcinoma; 27HC, 27-hydroxycholesterol; LXR, liver X receptor; NAFLD, nonalcoholic fatty liver disease; MDSC, myeloid-derived suppressor cell; PUFAs, polyunsaturated fatty acids; ROS, reactive oxygen species; SREBPs, sterol regulatory element binding proteins; TIMP1, tissue inhibitor of metalloproteinase-1; MMP, matrix metalloproteinase; $\mathrm{CD}$, cluster of differentiation; ERK, extracellular signal-regulated kinase; PI3K, phosphoinositide 3 kinase; Akt, protein kinase B; RhoA, Ras homolog family member A; TGF, transforming growth factor; FAK, focal adhesion kinase

Key words: fatty acid metabolism, fatty acid synthase, hallmarks of cancer, drug targets, cancer therapy, tumour microenvironment survival, proliferation, differentiation and metastasis of cancer cells. Several bodies of evidence have confirmed the metabolic reprogramming of FAs that occurs during cancer development. The present review aimed to evaluate FAs in terms of how the hallmarks of cancer are gradually established in tumourigenesis and tumour progression, and consider the auxo-action and exact mechanisms of FA metabolism in these processes. In addition, this interaction in the tumour microenvironment was also discussed. Based on the role of FA metabolism in tumour development, targeting FA metabolism may effectively target cancer, affecting a number of important characteristics of cancer progression and survival.

\section{Contents}

1. Introduction

2. FA metabolism and angiogenesis

3. FA metabolism and the activation of invasion and metastasis

4. FA metabolism and resisting cell death

5. FA metabolism and avoiding immune destruction

6. FA metabolism and enabling replicative immortality

7. FA metabolism and sustaining proliferative signalling

8. FA metabolism and evading growth suppressors

9. FA metabolism and the tumour microenvironment

10. Potential drug targets in cancer therapy

11. Concluding remarks

\section{Introduction}

In the course of human tumour pathogenesis, normal cells progressively acquire a sequence of biological abilities, known as the hallmarks of cancer, which comprise the six established hallmarks of sustaining proliferative signalling, evading growth suppressors, resisting cell death, enabling replicative immortality, inducing angiogenesis, and activating invasion and metastasis. There are also two emerging hallmarks, namely the programming of energy metabolism and evading immune destruction $(1,2)$. 
In order to meet the needs of biosynthesis during high levels of proliferation, the reprogramming of fatty acid (FA) metabolism becomes essential in cancer cells. At present, a number of previous studies have investigated the effects of cancer on FA metabolism during progression, demonstrating the increased de novo biogenesis and $\beta$-oxidation of FAs in these transformed cells. However, whether this influence is unilateral remains to be fully elucidated.

In the present review, evidence regarding the contribution of the change in FAs in cells during the acquisition and development of the hallmarks of cancer is discussed. In addition, the review provides a comprehensive insight into the underlying roles of FAs in tumourigenesis that go beyond their function in membrane phospholipid synthesis, signal transduction and energy production. Due to the diversity of cancer phenotypes and limitations of existing evidence, results on the associations may not be universal. However, the aim of the present review was to provide a diverse perspective in order to obtain a better understanding of the pathogenesis of cancer and to highlight potential novel treatment targets and therapies.

\section{FA metabolism and angiogenesis}

The induction of angiogenesis, including the sprouting of existing blood vessels and tubular arrangement of endothelial cells (ECs), is crucial for metastatic spread and for primary and metastatic tumour growth. The overstimulation of angiogenic growth by induced signals in tumour progression results in various tumour vasculature abnormalities (3), which in turn induces hypoxia, nutrient deficiency, reduced drug delivery, and more aggressive tumour growth (4); this forms a vicious circle of events. Tumour angiogenesis has long been recognised as an important target for anticancer therapy, and evaluation from the perspective of FA metabolism is an interesting area to evaluate first.

FA synthase (FASN), an enzyme associated with the endogenous synthesis of palmitic acid, is overexpressed invarious types of human cancer, and its expression level is associated with prognosis and invasion depth. Increased knowledge of its connection with angiogenesis is gradually being obtained.FASN can affect the expression of a series of vasculogenesis-related factors, including promotive growth-regulated protein family members, angiogenin and interleukin (IL)-6, and suppressants tissue inhibitor of metalloproteinase-1 (TIMP-1) and tissue inhibitor of TIMP-2 (5). It has been reported that FASN promotes angiogenesis in colorectal cancer by stimulating the secretion of angiogenic factors and the proliferation of ECs (5); significantly increased expression of FASN has also been positively correlated with elevated vascular endothelial growth factor (VEGF) levels (Fig. 1) (6). The significant impact of FASN inhibitors on angiogenesis has also been demonstrated in previous studies; by inhibiting the proliferation of ECs, they induce the inhibitory effect of angiogenesis (7). The same effect has also been observed in melanoma and glioma, supporting the key role of FASN in the neovascularization of tumours $(8,9)$.

The role of another factor closely associated with angiogenesis, FA oxidation (FAO), is gradually being elucidated. During vessel sprouting, FAO is involved in the growth and differentiation of vascular ECs (10). Although not imperative for the production of energy in ECs, FAO supplies carbon for glutamic acid to facilitate the synthesis of deoxyribonucleotides $(11,12)$; this is significant as the carbon source required for this synthesis is predominantly from glucose and glutamine (13). In addition, as the rate limiting enzyme of FAO, the pharmacological inhibition of carnitine palmitoyl transferase 1 (CPT1) or genetic loss of CPT1 in ECs can markedly reduce pathologic angiogenesis $(11,12)$.

FA-binding protein (FABP), an intracellular FA carrier protein, has also been observed to correlate with this hallmark of cancer. Elmasri et al (14) identified that FABP4 was closely associated with the proliferation and migration of ECs, in addition to vessel sprouting. Mechanically, a previous study suggested that FABP may be associated with increased VEGFA and neovascularization by binding with VEGF receptor 2 on the cell membrane (15). Kazlauskas (16) hypothesised that the expression of VEGF may be enhanced through mechanisms associated with FABP5 and peroxisome proliferator activated receptor $\gamma$ in prostate cancer cells following the uptake of exogenous FAs, indicating that there may be a link between the expression of VEGF and FABP. In addition, signal transduction lipids, including phenyl glycidyl ether 2 (PGE2) and lysophosphatidic acid, are important for the vascular growth and mobilisation of immunocytes, particularly macrophages that accelerate angiogenesis in tumours.

These results are indicative of an important association between FA metabolism and angiogenesis; they provide a basis for novel potential therapeutic strategies for targeting angiogenesis in cancer at different stages.

\section{FA metabolism and the activation of invasion and metastasis}

Several types of cancer gradually obtain the characteristics of invasion and metastasis with deterioration, an ability that is associated with the development and poor prognosis of cancer. Through an invasion-metastasis cascade, cancer cells infiltrate nearby blood vessels and lymphatic ducts, or are transferred to distant tissues (17). During this progression, an important mode is epithelial-mesenchymal transition (EMT). Through this process, cancer cells reverse to an undifferentiated state and possess the abilities of invasion, transmission and resistance to apoptosis. In this reversible process, the majority of enzymes associated with these epigenetic modifications require the involvement of cofactors including acetyl coenzyme A (acetyl-CoA), the intermediate products in FA metabolism, which causes EMT to become susceptible to the changing intracellular metabolite levels (18).

FA transporter [FAT; also known as cluster of differentiation (CD)-36], a membrane glycoprotein involved in transporting FAs, provides certain groups of cancer cells with unique metastasis-initiating potential and, particularly in human squamous cell carcinoma, CD36 has been identified as the inducer of distant metastasis $(19,20)$. CD36 is also involved with poor, long-term outcomes in several types of cancer, including melanoma and breast carcinoma, due to its prometastatic characteristics (19). The expression of FAT is linked with that of Wnt and transforming growth factor (TGF)- $\beta$ pathway-related genes, which are two potential activators of EMT (21). Nath et al (20) also demonstrated that the uptake of free FA (FFA), elevated by CD36, activated TGF- $\beta$ signalling pathways, which in turn activated EMT. Correspondingly, due to the correlation between CD36 and metastasis, the suppression of 


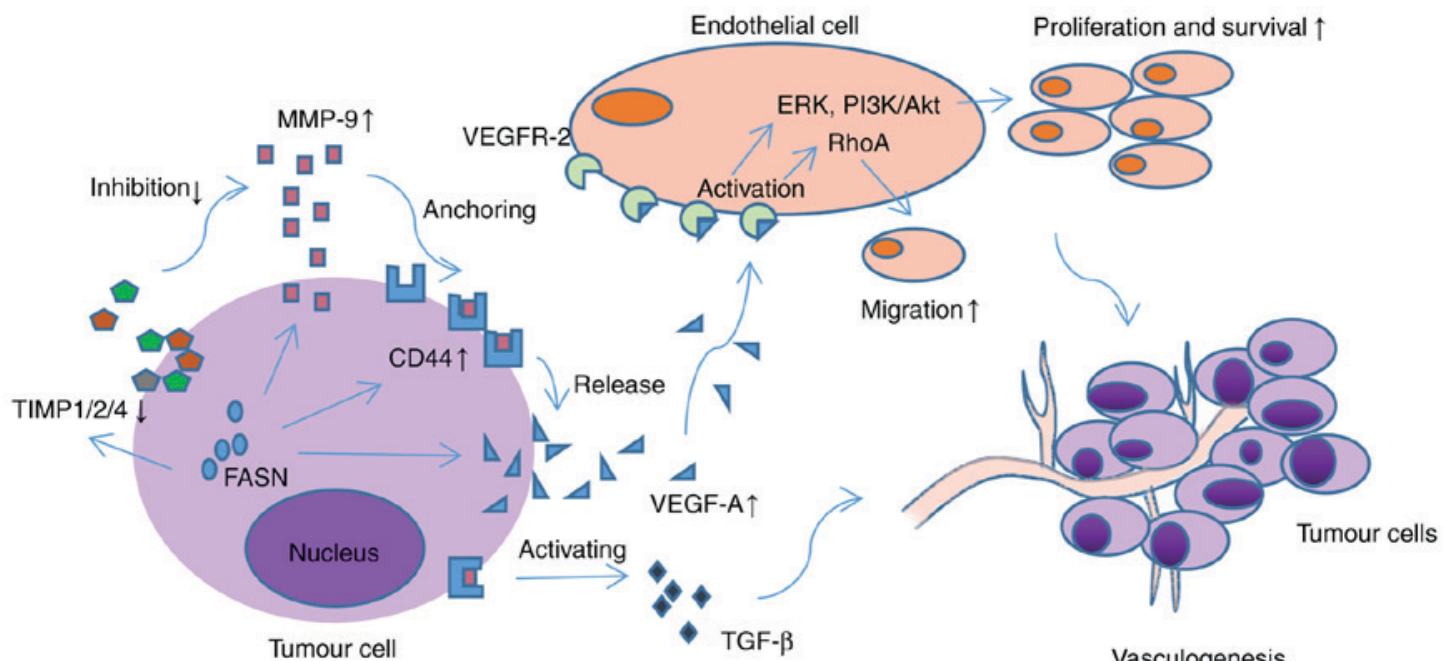

Figure 1. Mechanism by which FASN promotes angiogenesis through the regulation of VEGFA. By regulating the expression of TIMP1/2/4, MMP-9 and CD-44, FASN affects the synthesis and release of VEGFA, and the activation of VEGFR2. By activating the downstream ERK, PI3K/Akt and RhoA signalling pathways, the proliferation and migration of vascular endothelial cells and angiogenesis of the tumour are enhanced. In addition, the CD44/MMP9 complex can activate proangiogenic TGF- $\beta$ precursors and promote tumour vasculogenesis. FASN, fatty acid synthase; VEGFR, vascular endothelial growth factor receptor; TIMP, tissue inhibitor of metalloproteinase; MMP, matrix metalloproteinase; $\mathrm{CD}$, cluster of differentiation; ERK, extracellular signal-regulated kinase; PI3K, phosphoinositide 3 kinase; Akt, protein kinase B; TGF- $\beta$, transforming growth factor- $\beta$.

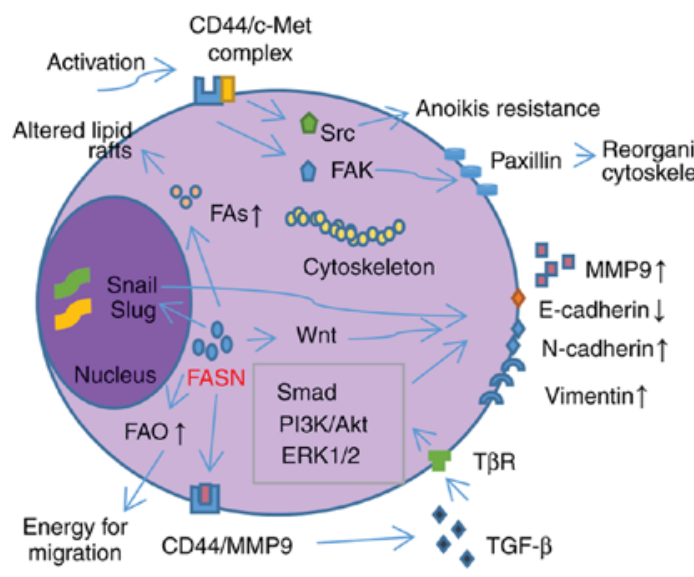

Tumour cell with epithelial phenotype

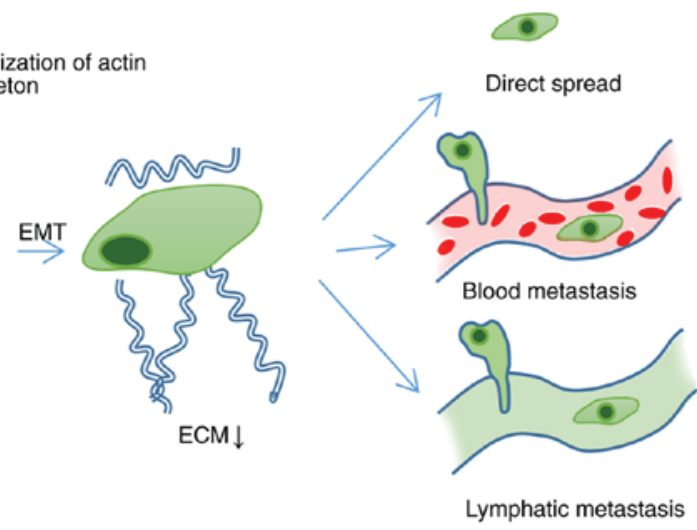

Mesenchymal phenotype

Figure 2. FASN promotes EMT conversion and the metastasis of tumour cells through various mechanisms. By promoting the synthesis of FAs, FASN alters the composition of lipid rafts and activates the CD44/c-Met complex, inducing the activation of Src, FAK and paxillin, and reorganisation of the actin cytoskeleton, resulting in changes in cell morphology and increased mobility. FASN can also regulate the expression of MMP9, E-cadherin, N-cadherin and Vimentin by activating the Wnt and TGF- $\beta$ signalling pathways, and promoting the expression of Snail and Slug transcription factors; this triggers the EMT of tumour cells. FAO can also be promoted to provide sufficient energy for tumour migration. FAs, fatty acids; FASN, fatty acid synthase; EMT, epithelial-mesenchymal transition; FAK, focal adhesion kinase; FAO, fatty acid oxidation; CD, cluster of differentiation; PI3K, phosphoinositide 3 kinase; Akt, protein kinase B; ERK, extracellular signal-regulated kinase; TGF- $\beta$, transforming growth factor- $\beta$.

CD36 also compromises the development of metastasis. A previous study suggested that inhibiting CD36 using neutralising antibodies resulted in a decrease in metastasis in mice with deficient immune systems (19). Furthermore, the induction of EMT requires complex remodelling of cellular lipid composition in order to alter the membrane fluidity required for cell migration (22), thereby highlighting the possibility of targeting membrane fluidity for the suppression of metastasis.

Considering the potential dependence of cells with a high energy demand following EMT on FA metabolism, several concerns have been raised regarding the correlation between FASN and EMT. Polyak and Weinberg (23) revealed that FASN was upregulated in EMT cells and, following silencing the expression of FASN using short hairpin RNAs, EMT reversal was induced. This upregulation has also been demonstrated in peritoneal metastasis of oophoroma; FASN was observed to promote EMT via the transcriptional regulation of different cadherins (24). Other studies have reported that FASN may exert its influence on invasion and metastasis by regulating the Wnt or TGF- $\beta$ signalling pathways (25). By promoting the synthesis of FAs, FASN alters the composition of lipid rafts and activates the CD44/c-Met complex, inducing the activation of Src, focal adhesion kinase and paxillin, in addition to reorganisation of the actin cytoskeleton, resulting in changes 
in cell morphology and increased mobility (Fig. 2) (26). In addition, the suppression of FASN was reported to be connected with the abrogation of EMT induced by hepatocyte growth factors, and reverse the poor differentiation and malignant characteristics in transformed cells $(27,28)$. Taken together, these results suggest that there may be a positive correlation between FASN and EMT.

Other enzymes associated with FA metabolism also have a number of associations with this translation. The overexpression of acetyl-CoA synthase and stearoyl-CoA desaturase-1 (SCD1) can activate EMT in colorectal cancer; however, acetyl-CoA synthase (ACS)2 acetylises hypoxia-inducible factor (HIF)-2 $\alpha$, one of the key inducers of EMT, to induce a prohibitive effect, and the overexpression of acyl-CoA medium-chain synthetase 3 also impairs the migration and invasion of hepatocellular carcinoma (HCC) cells $(29,30)$. In addition, a high expression level of adenosine triphosphate citrate lyase (ACL) is responsible for lymphatic metastasis in gastric adenocarcinoma, and silencing ACL reverses EMT in lung cancer accompanied by the downregulation of Snail, which can promote EMT in cancer $(31,32)$. Furthermore, acetyl-CoA carboxylase 2 silencing has a protective effect on the EMT transformation induced by glucose stress, triglyceride deposition and the accumulation of malonyl-CoA in the kidneys (33). Mitochondrial 3-hydroxy-3-methylglutaryl-CoA synthase, a key enzyme in lipid synthesis, also increases the metastatic potential of colorectal cancer cells by activating $\mathrm{Src}$ signalling (34).

In addition to these enzymes, FAs and the corresponding receptors are involved in the regulation of EMT. Elevated FFA levels can promote the metastatic progression of HCC via the transcriptional activation of EMT (20). Furthermore, a high expression level of FABP, which is responsible for the combination and transmission of long chain FAs, has been also reported to enhance metastasis in colorectal cancer (35).

The interactions in the tumour microenvironment are also key in this transformation. 27-Hydroxycholesterol, produced by tumour-associated macrophages or cholesterol metabolism, increases lung metastasis by acting through liver $\mathrm{X}$ receptor secondary to effects on EMT (36). Triglyceride catabolism in adipocytes drives the homing, migration and invasion of ovarian cancer cells by supplying FAs as energy substrates (37). In addition, adipose tissue-derived stem cells can be stimulated by breast cancer cells to secrete stromal cell-derived factor- 1 , and in turn, these paracrine factors enable cancer cells to migrate, invade and metastasise (38). In such a microenvironment, the actions of tumour cells are not isolated, but are the result of the combined effects of intracellular and extracellular resistance factors and attributing factors.

In addition, metabolism in cells with EMT is reprogrammed to meet energy requirements. Lipid metabolism can be regulated by EMT transcription factors, including the repressor of E-cadherin, which also affects the signal cascade required for the complete activation EMT $(39,40)$.

Therefore, taken together, the aforementioned evidence indicates that there are multiple associations between FA metabolism and the metastasis of cancer, which are associated with advanced stage, poor prognosis and malignant characteristics, including drug resistance. Furthermore, due to the interactions between the various factors that induce
EMT and feedback regulation, the complex signalling network for cell invasion and metastasis presents difficulties in the understanding, prevention and treatment of cancer. Further investigation into FA metastasis may assist in this understanding.

\section{FA metabolism and resisting cell death}

Apoptosis. Apoptosis, a mechanism that maintains homeostasis through programmed cell death controlled by genes, has long been considered the biggest challenge in the onset of cancer since it was first suggested by Kerr et al in 1972 (41). With regulation of different B-cell lymphoma 2 (Bcl-2) members, cells can initiate this program in response to DNA damage and insufficient growth factors (42). Correspondingly, cancer evades this cell death mechanism via p53 deletion, thereby lowering the expression of pro-apoptotic factors or increasing the expression of anti-apoptotic factors and survival signals. FA metabolism is also involved in this transformation.

ACL and FASN, important enzymes and the primary obstacles in FA synthesis, indirectly affect the development and apoptosis of cancer cells. Nishi et al (43) demonstrated that the use of 5-(tetradecyloxy)-2-furoic acid, an acetyl-CoA carboxylase inhibitor, increased the number of apoptotic cells by increasing the activity of caspase 3 , and the addition of palmitate attenuated this, suggesting that there may be a link between FA and apoptosis. The use of selective FASN inhibitors to promote apoptosis also supports this (44). Mechanically, Bandyopadhyay et al (45) reported that the increase in malonyl-CoA and the induction of pro-apoptotic genes tumour necrosis factor-related apoptosis-inducing ligand, Bcl-2-interacting protein 3 and death-associated protein kinase 2 , induced by the inhibition of FASN, may be the primary causes. It is also suggested that the accumulation of NADPH in this process may have a strong correlation with apoptosis (46). Therefore, there is a clear association between FA synthesis and apoptosis.

In addition, as expected, successful FAO is associated with the anti-apoptotic ability of cancer. Samudio et al (47) revealed that human leukaemia cells with inhibited FAO were more susceptible to apoptosis induced by ABT-737, an activator of $\mathrm{Bcl}-2$, resulting from the fact that $\mathrm{FAO}$ acts as a regulator of Bcl-2 antagonist/killer 1-dependent mitochondrial permeability. Furthermore, the main cause of the rapid accumulation of lipid droplets, one of the characteristics of apoptosis, is the suppression of FAO accompanied by an enhancement in the activity of acyl-CoA synthase (48). The cooperation of FA metabolism is an important strategy to consider in future investigations.

Autophagy. Autophagy, an important form of cell death, is a self-digestive process in which intracellular organelles or proteins are degraded in order to meet the needs of cell metabolism or the reprogramming of certain organelles. However, the role of autophagy in the development of tumours is complex and multifaceted. At present, autophagy is considered to function as a tumour suppressive process in the early stages of tumourigenesis by eliminating cytotoxic substrates, however, it also contributes to tumour cell resistance to apoptosis under 
energy stress conditions in established tumour autophagy (49). The triggering mechanism of autophagy in tumours is complicated, and FA metabolism is involved in this process.

As important products in FA synthesis, the accumulation of palmitic acids in cells can trigger autophagy through lipotoxic effects via the mammalian target of rapamycin (mTOR)-independent signalling pathway (50). In addition, FAs released by adipocytes in the tumour microenvironment can also trigger autophagy to promote cell survival in adverse environments via absorption by tumour cells and the activation of adenosine monophosphate-activated protein kinase (AMPK) signals (51). A novel hypothesis is that different types of FAs stimulate autophagic responses through distinct molecular mechanisms: Saturated FAs may trigger autophagy by activating the AMPK, protein kinase R, c-Jun N-terminal kinase 1 and lipid kinase complex, whereas unsaturated FAs may induce this process via an intact Golgi apparatus (52).

\section{FA metabolism and avoiding immune destruction}

The immune system is considered to be the greatest challenge for tumours to overcome in order to develop. The well-established theory of immune surveillance states that, through constant examination, the immune system is responsible for the recognition and elimination of nascent transformed cells via various means of monitoring, which is vital for prevention of the emergence, development and metastasis of cancer. However, through immunoediting processes, in which weak immunogenic tumour cells are allowed to evade and undergo further expansion while the immune system inhibits tumour growth (53), or the absence or inhibition of a link in the immune system, this mechanism is avoided and solid tumours form. In this process, FA metabolism leads to the promotion of immune evasion through effects in different sites.

Previous studies have demonstrated that FA metabolism is important in the development, differentiation, function and distribution of different $\mathrm{T}$ cell subsets (54); supporting the hypothesis that FA metabolism regulation has an effect on the normal function and failure of the immune system. Kleinfeld and Okada (55) reported that the cytotoxicity of $\mathrm{T}$ lymphocytes can be reduced by the FFA released by breast cancer tissues to evade immune injury. In addition, the accumulation of linoleic acid in non-alcoholic fatty liver disease is associated with the loss of $\mathrm{CD}^{+} \mathrm{T}$ cells via the induction of mitochondrial damage, leading to the occurrence of HCC (56). Myeloid-derived suppressor cells (MDSCs), associated with immune deficiency in cancer through the production of immunosuppressive cytokines and the inhibition of T lymphocytes, can be arrested by the pharmacological inhibition of FAO (57). A previous study also revealed that MDSCs with FA accumulation induced by the overexpression of fatty acid transport protein 4 have a higher prohibitive effect on immune cells than normal MDSCs (58). All of the above evidence is suggestive of a promotion of FA metabolism in the development of immune system evasion.

Alterations in FAs are also closely associated with the sensitivity of cancer cells to immune system-induced cell death. In humoral immunity, regulation of the biosynthesis of cell membrane FAs and lipids can affect the sensitivity of tumour cells to antibody-mediated lysis (59). In cellular immunity, Shaikh andEdidin (60) indicated that the sensitivity of target cells to effector $\mathrm{T}$ cells can be markedly reduced by the addition of polyunsaturated FAs; this effect is reported to arise during the identification phase (61). In addition, immunity against the cytotoxicity mediated by natural killer cells can be enhanced by an increase in oleic acid and linoleic acid in the membrane of HCC cells (62).

Macrophages, including tumour-prohibitive M1 type and tumour-promoting M2 type macrophages, are involved in the phagocytosis, antigen presentation and the secretion of cytokines and are important parts of the immune system. The M2 polarization of tumour-associated macrophages, an immunosuppressive phenotype, is known to be associated with an increase in FAO (63). Furthermore, the PGE2 released by cancer cells can transform tumour-associated macrophages from the tumour-suppressing M1 phenotype to the tumour-promoting M2 phenotype, resulting in immune system evasion (64). PGE2 can also inhibit the immune response in cancer by inducing the immunosuppressive cytokine IL-10 (65). As an important part of the immune system, functional changes in macrophages induced by FA metabolism are attributable to this evasion.

\section{FA metabolism and enabling replicative immortality}

In the ongoing replication process, the telomere length of normal cells gradually reduces and aging or cell death can be induced to a certain extent. Therefore, for the majority of cancer cells, this mechanism requires avoidance in order to permit infinite reproduction. In the regulation of the appearance of the senescence state, the formation and activity of telomerase is the most promising method. During this progression, FAs are directly or indirectly involved in the emergence of this important hallmark.

Cellular aging, an irreversible state in which the physiological function and proliferation and differentiation abilities gradually decline, is an important obstacle that requires avoidance by tumour cells in order to survive. Previous studies have suggested that the appearance of senescence is closely associated with the de novo synthesis and desaturation of FAs. The inhibition of FASN and SCD1, induced by telomere shortening and the subsequent activation of P53, results in increased levels of palmitic acid, reduced levels of monounsaturated FAs and phospholipids, and the induction of senescence $(66,67)$. Therefore, it is hypothesised that the modification of FA metabolism is vital in the aging process. In addition, carnitine palmitoyl transferase $1 \mathrm{C}$, an enzyme associated with the transportation and oxidation of FAs, is also associated with proliferation and senescence (68); therefore, altering the metabolism of FAs to delay or eliminate cell aging is beneficial for cancer progression.

The gradual reduction of telomeres, which protect chromosomal ends, is the greatest challenge for the immortal replication of cancer cells. Ponnusamy et al (69) demonstrated that the expression of FA elongase 3 is involved in determination of the length of telomeres. As the pivotal enzyme in lengthening telomeres, the activation of telomerase is also essential in the progression of cancer. Previous studies have revealed that polyunsaturated FAs (PUFAs), but not saturated FAs or trans-FAs, can significantly inhibit telomerase activity, 
and the enhancement of this effect is accompanied by an increase in the number of double bonds $(70,71)$. Furthermore, oleic acid, a monounsaturated FA, can competitively inhibit telomerase activity through its specific structure and molecular length (72).

The ability to duplicate without limit is essential for tumour development. Therefore, regulating FA metabolism or using unsaturated FAs to treat a wide variety of solid tumours and malignancies may be an effective strategy.

\section{FA metabolism and sustaining proliferative signalling}

The maintenance of proliferative signalling is a prerequisite for the formation, development and deterioration of tumours. Intracellular or extracellular ligands activate a sequence of signalling pathways, including the mitogen-activated protein kinase (MAPK) kinase/extracellular signal-regulated kinase (ERK) or phosphoinositide 3 kinase $(\mathrm{PI} 3 \mathrm{~K}) /$ protein kinase $\mathrm{B}$ (Akt)/mTOR signalling pathways, to have a regulatory function in the proliferation of transformed cells, mainly via the regulation of cell cycle. These mitogenic signals are controlled to produce, release, activate and degrade specific factors, thereby maintaining signalling balance and intracellular homeostasis. However, the abnormal secretion of ligands or constitutive activation of a signalling pathway, which FA metabolism is known to be involved in, can also result in the continuous proliferation of cancer cells (2).

Previous studies have indicated that the de novo synthesis of FAs is critical for completion of the cell cycle at the G2/M phase, further highlighting the potential of targeting FA metabolism to eradicate excessive proliferation (73). Of note, several phases of abnormal FA anabolism are closely associated with this hallmark of cancer. Cai and Tu (74) demonstrated that the upregulation of acetyl-CoA, which is important for the production of FAs, can facilitate cell entry into the cell cycle by promoting the acetylation of growth-related genomic proteins. SCD1, a key enzyme in catalysing saturated fatty acyl-CoA into its monounsaturated state, is associated with the activation of ERK1/2 and the expression of cell cycle-related genes (75). FABP4 has also been shown to be vital in the induction of Akt and MAPK signalling cascades in several malignancies, including breast cancer and oral squamous cell carcinoma (76). The pharmacological inhibition of FASN has also been confirmed to be closely associated with pro-proliferative pathways (77). In addition, most notably, the antiproliferative effects of omega-3 FAs are also as a result of the regulation of cell cycle (78).

\section{FA metabolism and evading growth suppressors}

In the process of tumour progression, in order to combat potent negative regulators, tumour cells must obtain the ability to evade growth inhibitive factors, which are mainly composed of proteins encoded by tumour suppressor genes, the inhibition mechanism and TGF signalling pathways (2). Through the absence of associated gene expression or the destruction of inhibitory programs, tumours are able to evade the reactions that are responsible for the response to internal and external signals and the subsequent regulation of proliferation, senescence and apoptosis.
P53 protein, which is expressed by the p53 gene, is an important established tumour suppressor and is an important growth inhibitive factor as it functions as a regulator of transcription and the cell cycle; the loss of expression or mutations in P53 are known to contribute to the development of several types of cancer (79). A number of experiments have demonstrated that p53 is also closely associated with the metabolism of FAs. Saadi et al (80) demonstrated that p53 is involved in mitophagy and inhibits the production of reactive oxygen species which favour lipid accumulation. In addition, wild-type p53 exerts a negative effect on transformed cells, whereas mutant p53 may lead to the development and deterioration of tumours. Through the activation of sterol regulatory element binding proteins (SREBPs) and the upregulation of cholesterol production, the growth of breast cancer cells is accelerated (81). Therefore, p53 is able to regulate lipid metabolism and respond to stressors.

\section{FA metabolism and the tumour microenvironment}

The tumour microenvironment is important for transformed cells. The effect of the microenvironment on tumour metabolism is an important factor to consider. Hypoxia, inflammation, and the metabolism of adjacent cells can affect the occurrence and development of cancer. In this process, FA metabolism is synergistically or negatively involved in the interactions between tumours and the microenvironment.

Hypoxia. Due to the rapid growth of cancer cells and uncontrolled angiogenesis, hypoxia represents a significant environmental state. Furuta et al (82) demonstrated that hypoxia upregulates the expression of SREBP-1, an important transcription factor in the anabolism of FAs, which in turn promotes breast cancer progression. The inhibition of hypoxia in FAO also facilitates the survival, proliferation and metastasis of cancer cells (83). In addition, the synergistic effect of hypoxia and FA metabolism has been verified. Zhang et al (84) reported that the PI3K/Akt-mediated crosstalk between SCD1 and HIF- $2 \alpha$ contributes to cancer progression. Therefore, the promotion of metabolic changes in FAs under hypoxic conditions may be an area of interest for future investigations into cancer development.

Inflammation. Cancer-associated inflammation, which is usually caused by the necrosis of tumour cells or oncogenic changes and the subsequent chronic stimulation induced by immune cells, is a favourable environment for the growth or malignant transformation of tumour cells (85); tumour cells are also able to tolerate cell necrosis for the increase in growth factors during the immune response. The production of mediators that contribute to inflammation are also associated with the metabolism of FAs.

It has been suggested that n-3 PUFAs can be transformed into anti-inflammatory molecules by lipoxygenase, whereasn-6 PUFAs are primarily converted into the pro-inflammatory form by cyclooxygenase (86). In addition to the inhibition of the arachidonic acid metabolism and influencing the expression of inflammatory genes, n-3 PUFAs can also produce mediators, known as resolvins, which possess anti-inflammatory properties (87). Fazio et al (88) demonstrated that the EMT stimulated by inflammation in colorectal cancer can be inhibited by 
eicosapentaenoic acid, an n-3 PUFA. In addition, n-3 PUFAs can reduce the activation of macrophage inflammasomes to have an inhibitory effect (89). Further investigations into the links between inflammation and metabolism and regulating FA metabolism to alter the carcinogenic inflammatory microenvironment are required to potentially develop novel cancer therapies.

Stromal cells. Cancer progression is associated with interactions between stromal and cancer cells. As an important part of the tumour microenvironment, stromal cells promote the proliferation and invasion of cancer cells by providing metabolic substrates or signalling molecules. A previous study on colon cancer revealed that transformed cells are able to absorb the FFAs released by surrounding adipocytes to activate FAO and autophagy in order to facilitate cancer development (51). Similar auxo-action has been confirmed in ovarian cancer (90). In addition, in terms of the changing modalities, exosomes derived from adipocytes are considered to be associated with the invasion of melanoma through FAO (91).

\section{Potential drug targets in cancer therapy}

Targeting abnormal tumour metabolism is an attractive potential avenue for future direct medicines. Due the importance of FA metabolism in protein modification, the synthesis of the cell membrane and the localization of oncogenic molecules, pharmacological inhibitors that target the key enzymes in these processes may be effective in future therapies.

The early generation of FASN inhibitors, including cerulenin and C75, are limited in their application, despite their confirmed induction of apoptosis in cancer cells, due to side effects associated with weight and appetite $(92,93)$. As an anti-obesity drug, orlistat has also been shown to exert inhibitory effects on lipid metabolism and a certain degree of tumour suppression; however, its poor selectivity and membrane permeability prevent it from being an ideal antineoplastic agent clinically $(94,95)$. In addition, several novel generations of molecules targeting FASN, including GSK837149A, TVB2640, and plant-derived polyphenols, are currently in development; among these, TVB2640 has now moved into the human trials phase (96-98). Therefore, the accurate and efficient inhibition of tumour lipid metabolism offers promise as a novel therapeutic strategy.

\section{Concluding remarks}

The previous few decades of experimental results have indicated that cancer is similar to a metabolic disease that involves disordered energy metabolism in tumour cells $(99,100)$. The abnormal metabolism of FAs is known to be crucial in cancer biology and pathology (101). In general, any limitations on the essential capabilities of cancer cells can have an inhibitory effect on tumourigenesis and tumour progression. However, due to the diversity of cancer types and characteristics, the same therapeutic method tends to have different effects between different types of cancer. Further investigations are required on the association between FA metabolism and the various hallmarks of different types of cancer in order to determine the angiogenesis tendency or how cancer invasion and metastasisis facilitated. Targeting the corresponding intermediate products of a signalling pathway may have a good response, which in turn may highlight novel strategies and potential therapies (102). This targeted treatment is likely to be more effective and have fewer toxic side effects towards normal tissues, therefore, non-toxic metabolic therapy may become the primary method for treating cancer in the future. Furthermore, due to the correlation between FAs and cancer, other sources of FAs, including dietary habits, obesity and hyperlipidaemia, also require consideration in addition to the regulation of metabolic processes $(103,104)$. In future precision medicine, FA metabolism offers significant potential. However, due to the diversity of tumour types, the flexibility of tumour metabolism and complex interactions in the tumour microenvironment, drugs or therapies that may be effective and appropriate for clinical treatments require further verification.

\section{Acknowledgements}

Not applicable.

\section{Funding}

The present study was supported by National Program on Key Research Project of China (grant no. 2016YFC0902700), the National Natural Science Foundation of China (grant nos. 81672672, 81572650, 81772891, 81502357 and 81621062), the Natural Science Foundation of Zhejiang Province (grant no. Q142114001) and the State Key Laboratory of Oral Diseases Special Funded Projects.

\section{Availability of data and materials}

Not applicable.

\section{Authors' contributions}

$\mathrm{X}-\mathrm{HY}$ and X-HR wrote the manuscript. Y-LT and X-HL were involved in the conception of the aims of the review, provided suggestions for the outline and structure of the manuscript, and performed the final corrections. All authors read and approved the final manuscript.

\section{Ethics approval and consent to participate}

Not applicable.

\section{Patient consent for publication}

Not applicable.

\section{Competing interests}

The authors declare that they have no competing interests.

\section{References}

1. Hanahan D and Weinberg RA: The hallmarks of cancer. Cell 100: 57-70, 2000. 
2. Hanahan D and Weinberg RA: Hallmarks of cancer: The next generation. Cell 144: 646-674, 2011.

3. Konerding MA, Fait E and Gaumann A: 3D microvascular architecture of pre-cancerous lesions and invasive carcinomas of the colon. Br J Cancer 84: 1354-1362, 2001

4. Mcintyre A and Harris AL: Metabolic and hypoxic adaptation to anti-angiogenic therapy: A target for induced essentiality. EMBO Mol Med 7: 368-379, 2015.

5. Zaytseva YY, Elliott VA, Rychahou P, Mustain WC, Kim JT, Valentino J, Gao T, O'Connor KL, Neltner JM, Lee EY, et al: Cancer cell-associated fatty acid synthase activates endothelial cells and promotes angiogenesis in colorectal cancer. Carcinogenesis 35: 1341-1351, 2014

6. Li J, Dong L, Wei D, Wang X, Zhang S and Hua L: Fatty acid synthase mediates the epithelial-mesenchymal transition of breast cancer cells. Int J Biol Sci 10: 171-180, 2014.

7. Browne CD, Hindmarsh EJ and Smith JW: Inhibition of endothelial cell proliferation and angiogenesis by orlistat, a fatty acid synthase inhibitor. FASEB J 20: 2027-2035, 2006.

8. Zhou Y, Jin G, Mi R, Zhang J, Zhang J, Xu H, Cheng S, Zhang Y, Song W and Liu F: Inhibition of fatty acid synthase suppresses neovascularization via regulating the expression of VEGF-A in glioma. J Cancer Res Clin Oncol 142: 2447-2459, 2016.

9. Seguin F, Carvalho MA, Bastos DC, Agostini M, Zecchin KG, Alvarez-Flores MP, Chudzinski-Tavassi AM, Coletta RD and Graner E: The fatty acid synthase inhibitor orlistat reduces experimental metastases and angiogenesis in B16-F10 melanomas. Br J Cancer 107: 977-987, 2012.

10. Folkman J: Role of angiogenesis in tumor growth and metastasis. Semin Oncol 29 (6 Suppl 16): S15-S18, 2002.

11. Cantelmo AR, Brajic A and Carmeliet P: Endothelial metabolism driving angiogenesis: Emerging concepts and principles. Cancer J 21: 244-249, 2015.

12. Schoors S, Bruning U, Missiaen R, Queiroz KC, Borgers G, Elia I, Zecchin A, Cantelmo AR, Christen S, Goveia J, et al: Fatty acid carbon is essential for dNTP synthesis in endothelial cells. Nature 520: 192-197, 2015.

13. DeBerardinis RJ, Lum JJ, Hatzivassiliou G and Thompson CB: The biology of cancer: Metabolic reprogramming fuels cell growth and proliferation. Cell Metab 7: 11-20, 2008.

14. Elmasri H, Ghelfi E, Yu C, Traphagen S, Cernadas M, Cao H, Shi GP, Plutzky J, Sahin M, Hotamisligil G and Cataltepe S: Endothelial cell-fatty acid binding protein 4 promotes angiogenesis: Role of stem cell factor/c-kit pathway. Angiogenesis 15: 457-468, 2012.

15. Ku CY, Liu YH, Lin HY, Lu SC and Lin JY: Liver fatty acid-binding protein (L-FABP) promotes cellular angiogenesis and migration in hepatocellular carcinoma. Oncotarget 7: 18229-18246, 2016.

16. Kazlauskas A: Lysophosphatidic acid contributes to angiogenic homeostasis. Exp Cell Res 333: 166-170, 2015.

17. Talmadge JE and Fidler IJ: AACR centennial series: The biology of cancer metastasis: Historical perspective. Cancer Res 70: 5649-5669, 2010.

18. Li L and Li W: Epithelial-mesenchymal transition in human cancer: Comprehensive reprogramming of metabolism, epigenetics, and differentiation. Pharmacol Ther 150: 33-46, 2015.

19. Pascual G, Avgustinova A, Mejetta S, Martín M, Castellanos A, Attolini CS, Berenguer A, Prats N, Toll A, Hueto JA, et al: Targeting metastasis-initiating cells through the fatty acid receptor CD36. Nature 541: 41, 2017.

20. Nath A, Li I, Roberts LR and Chan C: Elevated free fatty acid uptake via CD36 promotes epithelial-mesenchymal transition in hepatocellular carcinoma. Sci Rep 5: 14752, 2015.

21. Röhrig F and Schulze A: The multifaceted roles of fatty acid synthesis in cancer. Nat Rev Cancer 16: 732-749, 2016.

22. Zhao W, Prijic S, Urban BC, Tisza MJ, Zuo Y, Li L, Tan Z, Chen X, Mani SA and Chang JT: Candidate anti-metastasis drugs suppress the metastatic capacity of breast cancer cells by reducing membrane fluidity. Cancer Res 76: 2037-2049, 2016.

23. Polyak K and Weinberg RA: Transitions between epithelial and mesenchymal states: Acquisition of malignant and stem cell traits. Nat Rev Cancer 9: 265-273, 2009.

24. Jiang L, Wang H, Li J, Fang X, Pan H, Yuan X and Zhang P. Up-regulated FASN expression promotes transcoelomic metastasis of ovarian cancer cell through epithelial-mesenchymal transition. Int J Mol Sci 15: 11539-11554, 2014.
25. Jiang L, Xiao L, Sugiura H, Huang X, Ali A, Kuro-o M, Deberardinis RJ and Boothman DA: Metabolic reprogramming during TGF $\beta 1$-induced epithelial-to-mesenchymal transition. Oncogene 34: 3908-3916, 2015.

26. Zaytseva YY, Rychahou PG, Gulhati P, Elliott VA, Mustain WC, O'Connor K, Morris AJ, Sunkara M, Weiss HL, Lee EY and Evers BM: Inhibition of fatty acid synthase attenuates CD44-associated signaling and reduces metastasis in colorectal cancer. Cancer Res 72: 1504-1517, 2012.

27. Hung CM, Kuo DH, Chou CH, Su YC, Ho CT and Way TD: Osthole suppresses hepatocyte growth factor (HGF)-induced epithelial-mesenchymal transition via repression of the c-Met/Akt/mTOR pathway in human breast cancer cells. J Agric Food Chem 59: 9683-9690, 2011.

28. Gonzalezguerrico AM, Espinoza I, Schroeder B, Park CH, Kvp CM, Khurana A, Corominas-Faja B, Cuyàs E, Alarcón T, Kleer C, et al: Suppression of endogenous lipogenesis induces reversion of the malignant phenotype and normalized differentiation in breast cancer. Oncotarget 7: 71151-71168, 2016.

29. Ruan HY, Yang C, Tao XM, He J, Wang T, Wang H, Wang C, Jin GZ, Jin HJ and Qin WX: Downregulation of ACSM3 promotes metastasis and predicts poor prognosis in hepatocellular carcinoma. Am J Cancer Res 7: 543-553, 2017.

30. Sun L, Kong Y, Cao M, Zhou H, Li H, Cui Y, Fang F, Zhang W, Li J, Zhu X, et al: Decreased expression of acetyl-CoA synthase 2 promotes metastasis and predicts poor prognosis in hepatocellular carcinoma. Cancer Sci 108: 1338-1346, 2017.

31. Hanai JI, Doro N, Seth P and Sukhatme VP: ATP citrate lyase knockdown impacts cancer stem cells in vitro. Cell Death Dis 4: e696, 2013.

32. Hanai J, Doro N, Sasaki AT, Kobayashi S, Cantley LC, Seth P and Sukhatme VP: Inhibition of lung cancer growth: ATP citrate lyase knockdown and statin treatment leads to dual blockade of mitogen-activated protein kinase (MAPK) and phosphatidylinositol-3-kinase (PI3K)/AKT pathways. J Cell Physiol 227: 1709-1720, 2012.

33. Xu Y, Huang J, Xin W, Chen L, Zhao X, Lv Z, Liu Y and Wan Q: Lipid accumulation is ahead of epithelial-to-mesenchymal transition and therapeutic intervention by acetyl-CoA carboxylase 2 silence in diabetic nephropathy. Metabolism 63: 716-726, 2014.

34. Chen SW, Chou CT, Chang CC, Li YJ, Chen ST, Lin IC, Kok SH, Cheng SJ, Lee JJ, Wu TS, et al: HMGCS2 enhances invasion and metastasis via direct interaction with PPAR $\alpha$ to activate Src signaling in colorectal cancer and oral cancer. Oncotarget 8: 22460-22476, 2017.

35. Koichiro K, Shogo S, Chiaki K, Yuki K, Ke Y and Hiroshi F: High expression of fatty acid-binding protein 5 promotes cell growth and metastatic potential of colorectal cancer cells. FEBS Open Bio 6: 190-199, 2016.

36. Nelson ER, Wardell SE, Jasper JS, Park S, Suchindran S, Howe MK, Carver NJ, Pillai RV, Sullivan PM, Sondhi V, et al: 27-Hydroxycholesterol links hypercholesterolemia and breast cancer pathophysiology. Science 342: 1094-1098, 2013.

37. Nieman KM, Kenny HA, Penicka CV, Ladanyi A, Buell-Gutbrod R, Zillhardt MR, Romero IL, Carey MS, Mills GB, Hotamisligil GS, et al: Adipocytes promote ovarian cancer metastasis and provide energy for rapid tumor growth. Nat Med 17: 1498-1503, 2011

38. Muehlberg FL, Song YH, Krohn A, Pinilla SP, Droll LH,Leng X, Seidensticker M, Ricke J, Altman AM, Devarajan E, et al: Tissue-resident stem cells promote breast cancer growth and metastasis. Carcinogenesis 30: 589-597, 2009.

39. Puisieux A, Brabletz T and Caramel J: Oncogenic roles of EMT-inducing transcription factors. Nat Cell Biol 16: 488-494, 2014.

40. Sciacovelli M and Frezza C: Metabolic reprogramming and epithelial-to-mesenchymal transition in cancer. FEBS J 284: 3132-3144, 2017.

41. Kerr JF, Wyllie AH and Currie AR: Apoptosis: A basic biological phenomenon with wide-ranging implications in tissue kinetics. Br J Cancer 26: 239-257, 1972.

42. Adams JM and Cory S: Bcl-2-regulated apoptosis: Mechanism and therapeutic potential. Curr Opin Immunol 19: 488-496, 2007.

43. Nishi K, Suzuki K, Sawamoto J, Tokizawa Y, Iwase Y, Yumita $N$ and Ikeda T: Inhibition of fatty acid synthesis induces apoptosis of human pancreatic cancer cells. Anticancer Res 36: 4655-4660, 2016. 
44. Ventura R, Mordec K, Waszczuk J, Wang Z, Lai J, Fridlib M, Buckley D, Kemble G and Heuer TS: Inhibition of de novo palmitate synthesis by fatty acid synthase induces apoptosis in tumor cells by remodeling cell membranes, inhibiting signaling pathways, and reprogramming gene expression. EBioMedicine 2: 806-822, 2015.

45. Bandyopadhyay S, Zhan R, Wang Y, Pai SK, Hirota S, Hosobe S, Takano Y, Saito K, Furuta E, Iiizumi M, et al: Mechanism of apoptosis induced by the inhibition of fatty acid synthase in breast cancer cells. Cancer Res 66: 5934-5940, 2006

46. Cui Y, Xing P, Wang Y, Liu M, Qiu L, Ying G and Li B: NADPH accumulation is responsible for apoptosis in breast cancer cells induced by fatty acid synthase inhibition. Oncotarget 8 : 32576-32585, 2017

47. Samudio I, Harmancey R, Fiegl M, Kantarjian H, Konopleva M, Korchin B, Kaluarachchi K, Bornmann W, Duvvuri S, Taegtmeyer $\mathrm{H}$ and Andreeff M: Pharmacologic inhibition of fatty acid oxidation sensitizes human leukemia cells to apoptosis induction. J Clin Invest 120: 142-156, 2010.

48. Boren J and Brindle KM: Apoptosis-induced mitochondrial dysfunction causes cytoplasmic lipid droplet formation. Cell Death Differ 19: 1561-1570, 2012.

49. White E: Deconvoluting the context-dependent role for autophagy in cancer. Nat Rev Cancer 12: 401-410, 2012.

50. Jia SN, Lin C, Chen DF, Li AQ, Dai L, Zhang L, Zhao LL, Yang JS, Yang F and Yang WJ: The transcription factor $\mathrm{p} 8$ regulates autophagy in response to palmitic acid stress via a mammalian target of rapamycin (mTOR)-independent signaling pathway. J Biol Chem 291: 4462-4472, 2016.

51. Wen YA, Xing X, Harris JW, Zaytseva YY, Mitov MI, Napier DL, Weiss HL, Mark Evers B and Gao T: Adipocytes activate mitochondrial fatty acid oxidation and autophagy to promote tumor growth in colon cancer. Cell Death Dis 8: e2593, 2017.

52. Niso-Santano M, Malik SA, Pietrocola F, Bravo-San Pedro JM, Mariño G, Cianfanelli V, Ben-Younès A, Troncoso R, Markaki M, Sica V, et al: Unsaturated fatty acids induce non-canonical autophagy. EMBO J 34: 1025-1041, 2015.

53. Schreiber RD, Old LJ and Smyth MJ: Cancer immunoediting: Integrating immunity's roles in cancer suppression and promotion. Science 331: 1565-1570, 2011

54. Lochner M, Berod L and Sparwasser T: Fatty acid metabolism in the regulation of T cell function. Trends Immunol 36: 81-91, 2015

55. Kleinfeld AM and Okada C: Free fatty acid release from human breast cancer tissue inhibits cytotoxic T-lymphocyte-mediated killing. J Lipid Res 46: 1983-1990, 2005.

56. Ma C, Kesarwala AH, Eggert T, Medina-Echeverz J, Kleiner DE, Jin P, Stroncek DF, Terabe M, Kapoor V, ElGindi M, et al NAFLD causes selective CD4(+) T lymphocyte loss and promotes hepatocarcinogenesis. Nature 531: 253-257, 2016.

57. Hossain F, Al-Khami AA, Wyczechowska D, Hernandez C, Zheng L, Reiss K, Valle LD, Trillo-Tinoco J, Maj T, Zou W, et al: Inhibition of fatty acid oxidation modulates immunosuppressive functions of myeloid-derived suppressor cells and enhances cancer therapies. Cancer Immunol Res 3: 1236-1247, 2015.

58. Cao W and Gabrilovich D: Abstract 3649: Contribution of fatty acid accumulation to myeloid-derived suppressor cell function in cancer. Cancer Res 71: 3649, 2011.

59. Harris DT: Changes in plasma membrane phospholipids inhibit antibody-mediated lysis. Biochem Biophys Res Commun 417 231-236, 2011

60. Shaikh SR and Edidin M: Immunosuppressive effects of polyunsaturated fatty acids on antigen presentation by human leukocyte antigen class I molecules. J Lipid Res 48: 127-138, 2007.

61. Harris DT: Alterations in target cell membrane phospholipids alter T cell but not NK cell killing. Immunobiology 218: 21-27, 2013.

62. Yoo TJ, Kuo CY, Spector AA, Denning GM, Floyd R, Whiteaker S, Kim H, Kim J, Abbas M and Budd TW: Effect of fatty acid modification of cultured hepatoma cells on susceptibility to natural killer cells. Cancer Res 42: 3596-3600, 1982.

63. Nomura M, Liu J, Rovira II, Gonzalez-Hurtado E, Lee J, Wolfgang MJ and Finkel T: Fatty acid oxidation in macrophage polarization. Nat Immunol 17: 216-217, 2016.

64. Luan B, Yoon YS, Le LJ, Kaestner KH, Hedrick S and Montminy M: CREB pathway links PGE2 signaling with macrophage polarization. Proc Natl Acad Sci USA 112: 15642-15647, 2015.
65. Kalinski P: Regulation of immune responses by prostaglandin E2. J Immunol 188: 21-28, 2012.

66. Ford JH: Saturated fatty acid metabolism is key link between cell division, cancer, and senescence in cellular and whole organism aging. Age (Dordr) 32: 231-237, 2010.

67. Maeda M, Scaglia N and Igal RA: Regulation of fatty acid synthesis and Delta9-desaturation in senescence of human fibroblasts. Life Sci 84: 119-124, 2009.

68. Chen Y, Wang Y, Huang Y, Zeng H, Hu B, Guan L, Zhang H, $\mathrm{Yu}$ AM, Johnson $\mathrm{CH}$, Gonzalez FJ, et al: PPAR $\alpha$ regulates tumor cell proliferation and senescence via a novel target gene carnitine palmitoyltransferase 1C. Carcinogenesis 38: 474-483, 2017.

69. Ponnusamy S, Alderson NL, Hama H, Bielawski J, Jiang JC, Bhandari R, Snyder SH, Jazwinski SM and Ogretmen B Regulation of telomere length by fatty acid elongase 3 in yeast. Involvement of inositol phosphate metabolism and Ku70/80 function. J Biol Chem 283: 27514-27524, 2008.

70. Eitsuka T, Nakagawa K, Suzuki T and Miyazawa T: Polyunsaturated fatty acids inhibit telomerase activity in DLD-1 human colorectal adenocarcinoma cells: A dual mechanism approach. Biochim Biophys Acta 1737: 1-10, 2005.

71. Eitsuka T, Nakagawa K and Miyazawa T: Dual mechanisms for telomerase inhibition in DLD-1 human colorectal adenocarcinoma cells by polyunsaturated fatty acids. Biofactors 21: 19-21, 2004

72. Mizushina Y, Takeuchi T, Sugawara F and Yoshida H: Anti-cancer targeting telomerase inhibitors: $\beta$-rubromycin and oleic acid. Mini Rev Med Chem 12: 1135-1143, 2012.

73. Scaglia N, Tyekucheva S, Zadra G, Photopoulos C and Loda M: De novo fatty acid synthesis at the mitotic exit is required to complete cellular division. Cell Cycle 13: 859-868, 2014.

74. Cai L and Tu BP: Acetyl-CoA drives the transcriptional growth program in yeast. Cell Cycle 10: 3045-3046, 2011.

75. Mauvoisin D, Charfi C, Lounis AM, Rassart E and Mounier C: Decreasing stearoyl-CoA desaturase-1 expression inhibits $\beta$-catenin signaling in breast cancer cells. Cancer Sci 104: 36-42, 2013

76. Lee D, Wada K, Taniguchi Y, Al-Shareef H, Masuda T, Usami Y, Aikawa T, Okura M, Kamisaki Y and Kogo M Expression of fatty acid binding protein 4 is involved in the cell growth of oral squamous cell carcinoma. Oncol Rep 31: 1116-1120, 2014

77. Gao Y, Lin LP, Zhu CH, Chen Y, Hou YT and Ding J: Growth arrest induced by $\mathrm{C} 75$, A fatty acid synthase inhibitor, was partially modulated by p38 MAPK but not by p53 in human hepatocellular carcinoma. Cancer Biol Ther 5: 978-985, 2006.

78. Pan J, Zhou S, Xiang R, Zhao Z, Liu S, Ding N, Gong S, Lin Y, Li X, Bai X, et al: An $\Omega-3$ fatty acid desaturase-expressing gene attenuates prostate cancer proliferation by cell cycle regulation. Oncol Lett 13: 3717-3721, 2017.

79. Hollstein M, Sidransky D, Vogelstein B and Harris CC: p53 mutations in human cancers. Science 253: 49-53, 1991.

80. Saadi H, Seillier M and Carrier A: The stress protein TP53INP1 plays a tumor suppressive role by regulating metabolic homeostasis. Biochimie 118: 44-50, 2015.

81. Parrales A and Iwakuma T: p53 as a regulator of lipid metabolism in cancer. Int J Mol Sci 17: E2074, 2016.

82. Furuta E, Pai SK, Zhan R, Bandyopadhyay S, Watabe M, Mo YY, Hirota S, Hosobe S, Tsukada T, Miura K, et al: Fatty acid synthase gene is up-regulated by hypoxia via activation of Akt and sterol regulatory element binding protein-1. Cancer Res 68: 1003-1011,2008.

83. Huang D, Li T, Li X, Zhang L, Sun L, He X, Zhong X, Jia D, Song L, Semenza GL, et al: HIF-1-mediated suppression of acyl-CoA dehydrogenases and fatty acid oxidation is critical for cancer progression. Cell Rep 8: 1930-1942, 2014.

84. Zhang Y, Wang $H$, Zhang J, Lv J and Huang Y: Positive feedback loop and synergistic effects between hypoxia-inducible factor- $2 \alpha$ and stearoyl-CoA desaturase- 1 promote tumorigenesis in clear cell renal cell carcinoma. Cancer Sci 104: 416-422, 2013.

85. Mantovani A, Allavena P, Sica A and Balkwill F: Cancer-related inflammation. Nature 454: 436-444, 2008.

86. Patterson WL III and Georgel PT: Breaking the cycle: The role of omega- 3 polyunsaturated fatty acids in inflammation-driven cancers. Biochem Cell Biol 92: 321-328, 2014.

87. Calder PC: n-3 Polyunsaturated fatty acids, inflammation, and inflammatory diseases. Am J Clin Nutr 83 (Suppl 6): S1505-S1519, 2006. 
88. Fazio C, Piazzi G, Vitaglione P, Fogliano V, Munarini A, Prossomariti A, Milazzo M, D'Angelo L, Napolitano M, Chieco P, et al: Inflammation increases NOTCH1 activity via MMP9 and is counteracted by Eicosapentaenoic acid-free fatty acid in colon cancer cells. Sci Rep 6: 20670, 2016.

89. Williams-Bey Y, Boularan C, Vural A, Huang NN, Hwang IY, Shan-Shi C and Kehrl JH: Omega-3 free fatty acids suppress macrophage inflammasome activation by inhibiting NF- $\kappa \mathrm{B}$ activation and enhancing autophagy. PLoS One 9: e97957, 2014.

90. Hansen KJ and Houten BV: Investigating the metabolic relationship between ovarian cancer cells and adipocytes: The role of fatty acid beta-oxidation. Gynecol Oncol 137 (Suppl 1): S110, 2015.

91. Lazar I, Clement E, Dauvillier S, Milhas D, Ducoux-Petit M, LeGonidec S, Moro C, Soldan V, Dalle S, Balor S, et al: Adipocyte exosomes promote melanoma aggressiveness through fatty acid oxidation: A novel mechanism linking obesity and cancer. Cancer Res 76: 4051-4057, 2016.

92. Tennant DA, Durán RV and Gottlieb E: Targeting metabolic transformation for cancer therapy. Nat Rev Cancer 10: 267-277, 2010.

93. Loftus TM, Jaworsky DE, Frehywot GL, Townsend CA, Ronnett GV, Lane MD and Kuhajda FP: Reduced food intake and body weight in mice treated with fatty acid synthase inhibitors. Science 288: 2379-2381, 2000.

94. Kridel SJ, Axelrod F, Rozenkrantz N and Smith JW: Orlistat is a novel inhibitor of fatty acid synthase with antitumor activity. Cancer Res 64: 2070-2075, 2004.

95. Hoover HS, Blankman JL, Niessen S and Cravatt BF: Selectivity of inhibitors of endocannabinoid biosynthesis evaluated by activity-based protein profiling. Bioorg Med Chem Lett 18: 5838-5841, 2008
96. Puig T, Benhamu B, Turrado C, Relat J, Ortega-Gutierrez S, Casals G, Marrero PF, Haro D, Brunet J, Lopez-Rodriguez ML and Colomer R: Novel poliphenolic inhibitors of fatty acid synthase (FASN) have potential as anticancer agents. Cancer Res 68, 2008

97. Infante J, Patel M, Hoff DV, Brenner A, Rubino C, McCulloch W, Zhukova-Harrill V and Parsey M: 3LBA Initial report of a first-in-human study of the first-in-class fatty acid synthase (FASN) inhibitor, TVB-2640. Eur J Cancer 50 (Suppl 6): S195-S196, 2014.

98. Vázquez MJ, Leavens W, Liu R, Rodríguez B, Read M, Richards S, Winegar D and Domínguez JM: Discovery of GSK837149A, an inhibitor of human fatty acid synthase targeting the beta-ketoacyl reductase reaction. FEBS J 275: 1556-1567, 2008.

99. Linehan WM, Srinivasan R and Schmidt LS: The genetic basis of kidney cancer: A metabolic disease. Nat Rev Urol 7: 277-285, 2010 .

100. Wishart DS: Is cancer a genetic disease or a metabolic disease? EBioMedicine 2: 478-479, 2015

101. Currie E, Schulze A, Zechner R, Walther TC and Farese RV Jr: Cellular fatty acid metabolism and cancer. Cell Metab 18: 153-161, 2013.

102. Chen T and Li H: Fatty acid metabolism and prospects for targeted therapy of cancer. Eur J Lipid Sci Tec 119, 2017

103. Mariette G, Anne T, Pierre A, Clavel-Chapelon F and Nicole C: Dietary fat, fatty acid composition and risk of cancer. Eur J Lipid Sci Tec 107: 540-559, 2010

104. Balaban S, Lee LS, Schreuder M and Hoy AJ: Obesity and cancer progression: Is there a role of fatty acid metabolism? Biomed Res Int 2015: 274585, 2015. 